\title{
Performer Selection in Human Reliability Analysis: D numbers Approach
}

\author{
J. Zhao, Y. Deng
}

\section{Jie Zhao}

Institute of Fundamental and Frontier Science

University of Electronic Science and Technology of China, Chengdu

\section{Yong Deng*}

Institute of Fundamental and Frontier Science

University of Electronic Science and Technology of China, Chengdu

*Corresponding author: dengentropy@uestc.edu.cn

\begin{abstract}
Dependence assessment among human errors in human reliability analysis (HRA) is an significant issue. Many previous works discussed the factors influencing the dependence level but failed to discuss how these factors like "similarity of performers" determine the final result. In this paper, the influence of performers on HRA is focused, in addition, a new way of D numbers which is usually used to handle with the multiple criteria decision making $(\mathrm{MCDM})$ problems is introduced as well to determine the optimal performer. Experimental result demonstrates the validity of proposed methods in choosing the best performers with lowest the conditional human error probability (CHEP) under the same circumstance.
\end{abstract}

Keywords: Human reliability analysis (HRA), D numbers, D-S evidence theory, multiple criteria decision making (MCDM), nuclear power plant.

\section{Introduction}

Human error is an important factor to be considered in the design and risk assessment of large complex systems [71], such as nuclear power plant operation [1,28,66], air traffic control [27,44], grounding of oil tankers [37] and IIR filters [40]. Dependence analysis within human reliability analysis (HRA) refers to evaluating the influence of the failure of the operator to perform one task on the failure probabilities of subsequent tasks [51]. Dependence between two sequent tasks within human reliability analysis means if the preceding task fails, the failure probability of the following task is higher than the success [72]. Therefore, an appropriate assessment of dependence is crucial to avoid underestimation of the risk. In the existing methods, the result of dependence assessment is a Conditional Human Error Probability (CHEP), given failure on the preceding task $[5,72]$.

The most widely used method is the technique for human error rate prediction (THERP) dependence method $[26,50]$. THERP introduces five levels of dependence corresponding to dfferent values of CHEPs and it suggests some of the factors that may influence the dependence level. The THERP model refers to three main factors: "Closeness in Time" (CT), "Task Relatedness" (TR) and "Similarity of Performers" (SP). And the assessment requires considerable expert judgment on identifying which factor is important and on how these factors influence the dependence level. As a result, a highly subjective process that may be insufficient traceability and reproducibility would be inevitable.

To overcome this limitation, Decision Trees (DTs) method $[2,17]$ and Fuzzy Expert System (FES) $[38,39,72]$ have been introduced to extend the THERP dependence model. However, the influence of performer is partially ignored to some degree in the existing methods, which is critical factor determining the dependence level. In addition, it is inevitable to deal with uncertainty in the modeling the influence of performer. As a result, it is necessary to develop a new HRA 
math model. The purpose of this paper is to present a model and discuss how the differences of performers affect the final result as well as conditional human error probability (CHEP). The proposed method is based on D numbers [10] which is efficient to handle uncertainty multiple criteria decision making problem (MCDM) $[9,55,56]$.

The paper is organized as follows. In Section 2, some preliminaries are briefly introduced, including HRA, D-S evidence theory and D numbers. In Section 3, the proposed methodology in HRA is detailed. In Section 4, an application of the proposed HRA method in post initiator HFEs of a nuclear power plant is used to illustrate the effectiveness of the presented method. Conclusion is given in Section 5 .

\section{Preliminaries}

In this section, some preliminaries including human reliability analysis (HRA), evidence theory and D numbers, are briefly introduced.

Definition 1. Assume that task $T_{B}$ is subsequent to task $T_{A}$, and $B$ and $A$ are the corresponding failure events. $P_{A}$ and $P_{B}$ are basic probability of failure of task $T_{A}$ and $T_{B}$, respectively, then the conditional human error probability (CHEP) of B given is defined as follows: [47]

$$
P_{X D}(B \mid A)=\left(1+K \times P_{B}\right) /(K+1)
$$

where $K=0,1,6,9, \infty$, for dependence levels $\mathrm{CD}, \mathrm{HD}, \mathrm{MD}, \mathrm{LD}$ and $\mathrm{ZD}$, where $\mathrm{XD}=\mathrm{CD}, \mathrm{HD}$, $\mathrm{MD}, \mathrm{LD}$ and $\mathrm{ZD}$, respectively. It reflects the less dependent the two tasks are, the lower the failure of probability is.

\section{$2.1 \quad$ D-S evidence theory}

It's necessary to deal with uncertainty $[34,35,63,65]$ and many math models are presented such as fuzzy set $[12,41,53,64]$, Z-numbers [24,25,59], belief function [20,60,61] and bio-inspired model [62]. Due to its efficiency to model uncertainty, evidence theory is widely used in decision making [3,14], pattern recognition [4,6,19] evidential reasoning [29,67-69] and information fusion $[46,58]$. Some basic definitions of D-S theory are briefly introduced $[7,42]$ :

Definition 2. A set of hypotheses $\Theta$ is the exhaustive hypotheses of variable and the elements are mutually exclusive in $\Theta$. Then $\Theta$ is called the frame of discernment, defined as follows $[7,42]$ :

$$
\Theta=\left\{H_{1}, H_{2}, \cdots, H_{i}, \cdots, H_{N}\right\}
$$

The power set of $\Theta$ is denoted by $2^{\Theta}$, and

$$
2^{\Theta}=\left\{\emptyset,\left\{H_{1}\right\}, \cdots,\left\{H_{N}\right\},\left\{H_{1}, H_{2}\right\}, \cdots,\left\{H_{1}, H_{2}, \cdots, H_{i}\right\}, \cdots, \Theta\right\}
$$

where $\emptyset$ is an empty set.

Definition 3. A BPA function $m$ is a mapping of $2^{\Theta}$ to a probability interval $[0,1]$, formally defined by $[7,42]$ :

$$
m: \quad 2^{\Theta} \rightarrow[0,1]
$$

which satisfies the following conditions:

$$
m(\emptyset)=0 \quad \sum_{A \in 2^{\Theta}} m(A)=1 \quad 0 \leq m(A) \leq 1 \quad A \in 2^{\Theta}
$$


The mass $m(A)$ represents how strongly the evidence supports $A$ with the efficiency to model uncertainty [9]. Conflicting management with Dempster rule is still an open issue [52]. In addition, many operations on mass function have been proposed such correlation [21], distance [11], entropy measure [31,36], divergence measure [13,45] and negation $[15,16]$.

For the same evidence, the different BPAs come from the different evidence resources. The Dempster's combination rule can be used to obtain the combined evidence [7]:

$$
\left\{\begin{array}{l}
m(\emptyset)=0 \\
m(A)=\frac{\sum_{B \cap C=A} m_{1}(B) m_{2}(C)}{1-K}
\end{array}\right.
$$

where $K=\sum_{B \cap C=\emptyset} m_{1}(B) m_{2}(C)$. It should be mentioned that many methods are presented to deal with the open issues of evidence theory.

Definition 4. Let $m$ be a BPA on $\Theta$. Its associated pignistic probability function Bet $P_{m}$ : $\Theta \rightarrow[0,1]$ is defined as:

$$
\operatorname{Betp}_{m}(\omega)=\sum_{A \in \Theta, \omega \in A} \frac{1}{|A|} \frac{m_{A}}{\left.1-m_{(} \emptyset\right)}, m(\emptyset) \neq 1
$$

where $|A|$ is the cardinality of subset $\mathrm{A}$.

\subsection{D numbers}

The real application exists the uncertainty [22,57]. To address the Multiple criteria decision making problem (MCDM) issue [23], a new mathematical tool called D numbers [10] to represent uncertain information is proposed. The D numbers overcome several limitations the D-S theory involved.

Definition 5. Let $\Omega$ be a finite nonempty set, a $\mathrm{D}$ number is a mapping that $\mathrm{D}: \Omega \rightarrow[0,1]$,with

$$
\sum_{A \in \Omega} D(A) \leq 1 \quad \text { and } \quad D(\emptyset)=0
$$

where $\emptyset$ is an empty set and A is a subset of $\Omega[10]$.

Besides the empty set is not necessary set ZERO [48,49], it should be pointed out that different from D-S theory, the elements in set $\omega$ do not require mutually exclusive and the completeness constraint is not necessary in D numbers. If $\sum_{A \in \Omega} D(A) \leq 1$, the information is said to be complete; Otherwise, the information is assumed to be incomplete.

For a discrete set $\Omega=\left\{b_{1}, b_{2}, \cdots, b_{i}, b_{j}, \cdots, b_{n}\right\}$, where $b_{i} \in \mathrm{R}$ and $b_{i} \neq b_{j}$, when $i \neq j$, a special form of $\mathrm{D}$ numbers can be expressed by

$$
\begin{array}{r}
D\left(b_{1}\right)=v_{1} \\
D\left(b_{2}\right)=v_{2} \\
\\
\cdots \\
D\left(b_{i}\right)=v_{i} \\
D\left(b_{j}\right)=v_{j} \\
\cdots \\
D\left(b_{n}\right)=v_{n}
\end{array}
$$


simply denoted as $\mathrm{D}=\left\{\left(b_{1}, v_{1}\right),\left(b_{2}, v_{2}\right), \cdots,\left(b_{i}, v_{i}\right),\left(b_{j}, v_{j}\right), \cdots,\left(b_{n}, v_{n}\right)\right\}$, where $v_{i}>0$ and $\sum_{i=1}^{n} v_{i} \leq$ 1. If $\sum_{i=1}^{n} v_{i}=1$, the information is said to be complete; If $\sum_{i=1}^{n} v_{i}<1$, the information is said to be incomplete. It is effective and convenient that using the form of $\mathrm{D}$ numbers to express the uncertain information in the real world.

Definition 6. Let $D_{1}=\left\{\left(b_{1}^{1}, v_{1}^{1}\right), \cdots,\left(b_{i}^{1}, v_{i}^{1}\right), \cdots,\left(b_{n}^{1}, v_{n}^{1}\right)\right\}, D_{2}=\left\{\left(b_{1}^{2}, v_{1}^{2}\right), \cdots,\left(b_{j}^{2}, v_{j}^{2}\right), \cdots\right.$ ,$\left.\left(b_{m}^{2}, v_{m}^{2}\right)\right\}$ be two $\mathrm{D}$ numbers, the combination of $D_{1}$ and $D_{2}$, indicated by $D=D_{1} \oplus D_{2}$, is defined by

$$
D(b)=v
$$

with

$$
\begin{gathered}
b=\frac{b_{i}^{1}+b_{j}^{2}}{2} \\
v=\frac{v_{i}^{1}+v_{j}^{2}}{2} / C \\
C=\left\{\begin{array}{llll}
\sum_{j=1}^{m} \sum_{i=1}^{n}\left(\frac{v_{i}^{1}+v_{j}^{2}}{2}\right), & \sum_{i=1}^{n} v_{i}^{1}=1 \quad \text { and } & \sum_{j=1}^{m} v_{j}^{2}=1 ; \\
\sum_{j=1}^{m} \sum_{i=1}^{n}\left(\frac{v_{i}^{1}+v_{j}^{2}}{2}\right)+\sum_{j=1}^{m}\left(\frac{v_{c}^{1}+v_{j}^{2}}{2}\right), & \sum_{i=1}^{n} v_{i}^{1}<1 \quad \text { and } & \sum_{j=1}^{m} v_{j}^{2}=1 ; \\
\sum_{j=1}^{m} \sum_{i=1}^{n}\left(\frac{v_{i}^{1}+v_{j}^{2}}{2}\right)+\sum_{j=1}^{m}\left(\frac{v_{i}^{1}+v_{c}^{2}}{2}\right), & \sum_{i=1}^{n} v_{i}^{1}=1 \quad \text { and } & \sum_{j=1}^{m} v_{j}^{2}<1 ; \\
\sum_{j=1}^{m} \sum_{i=1}^{n}\left(\frac{v_{i}^{1}+v_{j}^{2}}{2}\right)+\sum_{j=1}^{m}\left(\frac{v_{c}^{1}+v_{j}^{2}}{2}\right) & & \\
+\sum_{j=1}^{m}\left(\frac{v_{i}^{1}+v_{c}^{2}}{2}\right)+\frac{v_{c}^{1}+v_{c}^{2}}{2}, & \sum_{i=1}^{n} v_{i}^{1}<1 \quad \text { and } \quad & \sum_{j=1}^{m} v_{j}^{2}<1 ;
\end{array}\right.
\end{gathered}
$$

where $v_{c}^{1}=1-\sum_{i=1}^{n} v_{i}^{1}$ and $v_{c}^{2}=1-\sum_{j=1}^{n} v_{j}^{2}$

In the meanwhile, an aggregation operator is proposed on this special D number, it is defined as below.

Definition 7. Let $D=\left\{\left(b_{1}, v_{1}\right),\left(b_{2}, v_{2}\right), \cdots,\left(b_{i}, v_{i}\right), \cdots,\left(b_{n}, v_{n}\right)\right\}$ be a D number, the integration representation of $\mathrm{D}$ number is defined as:

$$
I(D)=\sum_{i=1}^{n} b_{i} v_{i}
$$

\section{The proposed method}

The motivation behind the development of the proposed method is to select best performers which is the key factor in HRA among alternatives using D numbers proposed by Deng [10]. The influence of performers should be paid more attention. After figuring out the best performers 


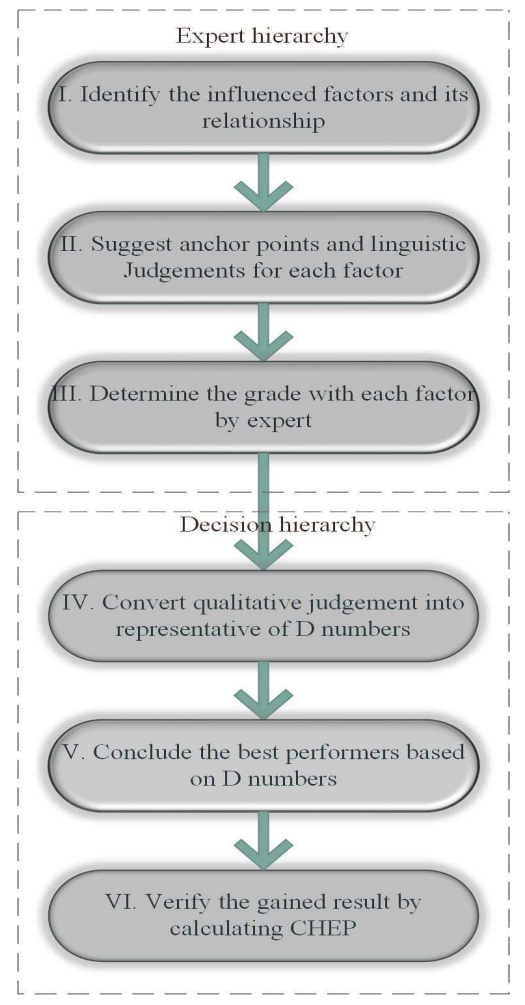

Figure 1: Framework of the proposed method

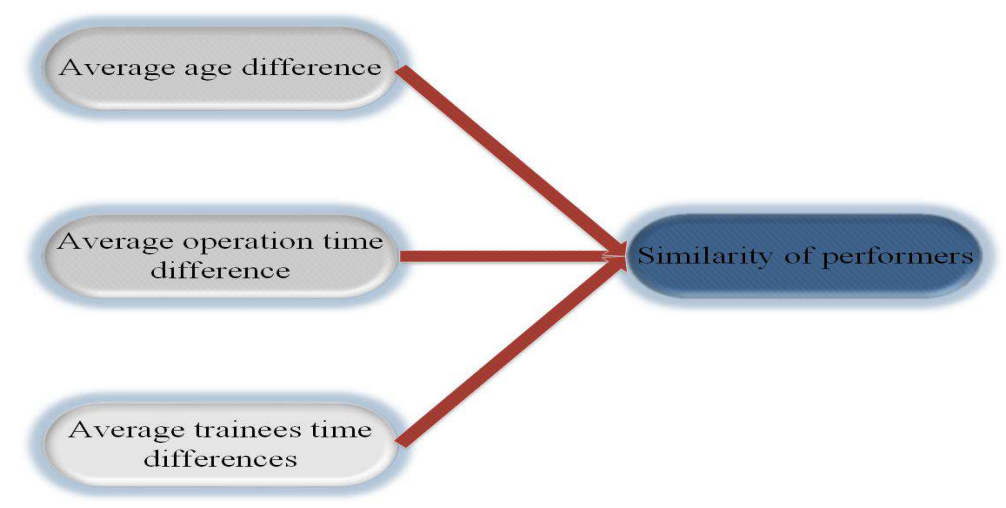

Figure 2: The factor affecting the performers

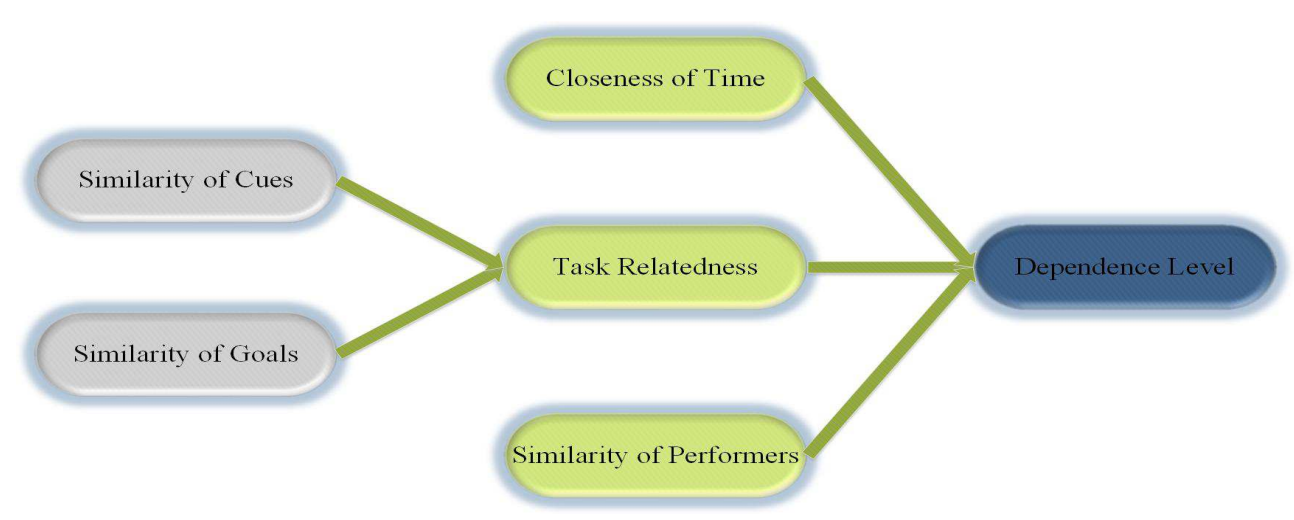

Figure 3: Functional relationships among the input factors of the dependence level 
based on D numbers, the result through calculating the CHEP will be verified. The flow chart is shown in Fig. 1.

Step 1. Identify the influenced factors and the functional relationship among them: The first step is to determine the factors that may have influence on the dependence between two human failure events. For example, the THERP [72] model refer to five main factors: "closeness in time", "task relatedness", "similarity of performers", "similarity of cues" and "similarity of goals" .And as for the performers, the influential factors the similarity of performers could be divided into three: "the average age among performers", "the average trainees time among performers" and "average operation time on the same task among performers". The relationship is shown in Fig. 2 and Fig. 3.

Step 2. Suggest anchor points and linguistic judgements for each factor: anchor points and linguistic judgements are provided by domain experts in advance as guidance for the HRA analyst's judgement of the input factors. Furthermore, due to the frequent ignorance of performers, the influential factors "the average age among performers", "the average trainees time among performers" and "average operation time on the same task among performers" are proposed. Their anchor points are shown in Tables (1-3). And a set of anchor points and linguistic judgements for the input factor SP is presented in Table 4, with the associated levels of dependence of performers. Dependence level of an input factors indicates the dependence level between two tasks with respect to this factor. For example, dependence level "ZD" in Table 4 means that the dependence level between two tasks is zero dependence with respect to the factor "similarity of performers".

\begin{tabular}{|c|c|c|}
\hline "Average operation time difference" anchor points & Linguistic judgement & Dep. level \\
\hline Less than $30 \mathrm{~min}$ & The tasks are accomplished by the same individual in operation time & $\mathrm{CD}$ \\
\hline More than $30 \mathrm{~min}$ but less than $1 \mathrm{~h}$ & High level of performer similarity in operation time exists & $\mathrm{HD}$ \\
\hline More than $1 \mathrm{~h}$ but less than $1.5 \mathrm{~h}$ & The level of performer similarity in operation time is medium & MD \\
\hline More than $1.5 \mathrm{~h}$ but less than $2 \mathrm{~h}$ & A low level of performers similarity in operation time exists & HD \\
\hline More than $2 \mathrm{~h}$ & NO similarity of performers in operation time is present between tasks & ZD \\
\hline
\end{tabular}

Table 1: Anchor points for input factor "average operation time difference"

\begin{tabular}{cc}
\hline "Average age difference "anchor points & Linguistic judgement \\
\hline less than 1 year & The age of the two performers are basically the same \\
more than 1 year but less than 3 years & The age similarity of two performers are almost the same \\
more than 3 years but less than 5years & The age level of performers similarity is medium \\
more than 5 years but less than 7years & The difference of the two performers are quite different in age \\
more than 7 years & The tasks are accomplished by the completely different individuals \\
\hline
\end{tabular}

Table 2: Anchor points for input factor "average age difference"

\begin{tabular}{ccc}
\hline "Average trainees time difference" anchor points & Linguistic judgement & Dep. level \\
\hline less than 1 year & The operating technology between two performers is basically same & CD \\
more than1 year but less than 1.5 year & The technology difference between two performers are slight & HD \\
more than 1.5 years but less than 2 years & The level of operating technology between two performers is medium & MD \\
more than 2 years but less than 2.5 years & The operating technology between two performers is basically different & HD \\
more than 2.5 years & The technology of the two teams are different & ZD \\
\hline
\end{tabular}

Table 3: Anchor points for input factor "average trainees time difference"

Step 3. Determine the grade with each factor by expert: It is supposed that the expert is totally authoritative. According to the collected data, the expert is asked to give their qualified judgement on each factor. The factor need to be judged by the expert are: the average age among performers, the average trainees time among performers, average operation time on the same task among performers, "closeness in time", "task relatedness". Due to our purpose is to verify best performers, the factors "closeness in time", "task relatedness" are set as constant. 


\begin{tabular}{|c|c|c|}
\hline Anchor points & Linguistic judgement & Dep. level \\
\hline TSC vs control shift room & NO similarity of performers is present between tasks & ZD \\
\hline Different team & A low level of performer similarity exist & $\mathrm{LD}$ \\
\hline Different individuals with same qualification & The level of performer similarity is medium & MD \\
\hline Same team & High level of performer similarity is present between tasks & HD \\
\hline Same person & The tasks are accomplished by the same individual & $\mathrm{CD}$ \\
\hline
\end{tabular}

Table 4: Judgement on factors given by experts after transformation

Step 4. Convert qualitative judgement into representation of D numbers: In the previous work in the HRA, the lack of objectivity is always a severe problem. The highlight of this paper is the representation of the expert's judgement, by which could effectively represent the judgement in an reasonable way as well as offer a new way to deal with Multiple criteria decision making problem (MCDM). The transformation from the judgement into the D numbers is shown in Eq. (9).

Take the "the average age among performers" of performer A as an example:

$D_{\text {age }}^{A}=\{(H D, 0.6),(C D, 0.4)\}$.

Step 5. Conclude the best performers based on D numbers: given the existed judgement, we can calculate the utility of each factor using Eqs. (8-13). Firstly aggregating the three influential factor one by one, then the utility of performer is obtained.

Take the performer A as an example:

$D_{\text {age }}^{A}=\{(H D, 0.6),(C D, 0.4)\}$

$D_{\text {ope }}^{A}=\{(H D, 0.3),(C D, 0.7)\}$

$D_{\text {time }}^{A}=\{(C D, 1.0)\}$

To simplify the representation and calculation, it translates the qualitative judgement into numerical form: the dependence level CD, HD, MD, LD, ZD are corresponding to 5, 4, 3, 2, 1 respectively. The rest of the paper would follow this simplification.

$D_{\text {age }}^{A}=\{(4,0.6),(5,0.4)\}$

$D_{\text {ope }}^{A}=\{(4,0.3),(5,0.7)\}$

$D_{\text {time }}^{A}=\{(5,1.0)\}$

According to Eqs. (9-12), the integrated D numbers by fusing above three D numbers is obtained:

$D_{\text {performer } A}=D_{\text {age }}^{A} \oplus D_{\text {ope }}^{A} \oplus D_{\text {time }}^{A}$

$D_{\text {performer } A}=\left\{\left(4.5, \frac{4.9}{16}\right),\left(4.75, \frac{6}{16}\right),\left(5, \frac{5.1}{16}\right)\right\}$

Then using Eq. (14), it can conclude:

$I\left(D_{\text {performer } A}\right)=5 \times \frac{5.1}{16}+4.75 \times \frac{6}{16}+4.5 \times \frac{4.9}{16}=4.7531$

In the transformation from expert's judgements into D numbers, the more dependent the influential factors of SP are, the higher value the D numbers would be. Therefore, it is could be said that by comparing the $\mathrm{I}(\mathrm{D})$ of each factor, the smaller number of $\mathrm{I}(\mathrm{D})$ is, the better performer is. Then the best performer is obtained.

In this paper, a transformation form D number to BPA is defined. Assuming there are several D numbers of one individual, the translation from D numbers into BPA is defined as:

$$
\begin{aligned}
& D_{i}^{1}=\left\{\left(b_{1}, v_{1}\right),\left(b_{2}, v_{2}\right)\right\} \\
& D_{j}^{1}=\left\{\left(b_{1}, v_{3}\right),\left(b_{2}, v_{4}\right)\right\} \\
& D_{k}^{1}=\left\{\left(b_{1}, v_{5}\right),\left(b_{2}, v_{6}\right)\right\}
\end{aligned}
$$

the BPA is obtained: 


$$
m\left(b_{1}\right)=\frac{v_{1}+v_{3}+v_{5}}{n}, m\left(b_{2}\right)=\frac{v_{2}+v_{4}+v_{6}}{n}
$$

where $n$ is the number of $\mathrm{D}$ numbers.

Step 6. verify the gained result by calculating CHEP: taking advantage of above process, the rank of alternative performers is obtained. In this paper, using Eqs. (15-16), the BPA of performers is obtained. Combining with the other two factors, "closeness in time" and "task relatedness", which are constant in order to control variables, to calculate the CHEP using Eqs. (1-7).

Take the above numerical data of performer A as an example. according to the Eqs. (15-16), the BPA of performer A is obtained:

$m_{\text {performer }}(5)=0.7, m_{\text {performer }}(4)=0.3$

Then assume

$m_{\text {time }}(5)=0.2, m_{\text {time }}(5)=0.6, m_{\text {time }}(5)=0.2$

$m_{\text {task }}(5)=0.2, m_{\text {task }}(4)=0.5, m_{\text {task }}(3)=0.3$

Thus according to the Eq. (5), the combination result $m_{\text {performer }} \bigoplus m_{\text {time }} \bigoplus m_{\text {task }}$ is:

$m_{\text {performer } A}(5)=m_{\text {performer }}(5) \bigoplus m_{\text {time }}(5) \bigoplus m_{\text {task }}(5)=0.2373$

$m_{\text {performer } A}(4)=m_{\text {performer }}(4) \bigoplus m_{\text {time }}(4) \bigoplus m_{\text {task }}(4)=0.7626$

According to the Eq. (7), we can conclude $\operatorname{Betp}(X D)=m(X D)$, because the used data is single subset.

where $\mathrm{XD}=\mathrm{CD}, \mathrm{HD}, \mathrm{MD}, \mathrm{LD}$, and $\mathrm{ZD}$.

Finally, the conditional human error probability (CHEP) $P(B \mid A)$ is calculated using Eq. (1) as: $P(B \mid A)=\sum_{X D} \operatorname{Bet} P(X D) \times P_{X D}(B \mid A)=0.2373 \times \frac{1+0.01 \times 0}{1+0}+0.7626 \times \frac{1+0.01 \times 1}{1+1}=0.6224$ Assume that the failed probability of task B is 0.01 . Given the previous failed task A, the reason why the failed probability of later task B is very high up to 0.6224 is that the relationship between the two successive tasks is complete dependent or highly dependent.

\section{Application}

In this section, a working model for post initiator HFEs of a nuclear power plant is used to illustrate the whole procedure of the proposed method. Furthermore, the working model of $\mathrm{D}$ numbers is used to determine the best performers that is a kind of multiple criteria decision making (MCDM). Then the traditional method in HRA is used to verify the proposed working model. At the end of this section, the effect of D numbers and its relationship with HRA will be discussed.

\subsection{Selection of best performers}

The identified input factors of interest and their relationship in the working model are shown in Fig. 3. According to the THERP method, three influential factors ("the average age difference", "the average trainees time difference" and "the average operation time in solving the same problem") directly affect the performers, which is the one of influential factor to dependence level. These factors are not necessarily relevant, but the model has enough complexity to illustrate the application of the methodology.

For each influential factor, anchor points and linguistic judgements corresponding to factor are provided by experts, as shown in Tables (1-4). In this paper, in order to figure out the best performer, D numbers is used as the representation of judgement offered by experts.

The representation of judgement in D numbers mainly depends on the number of experts who are in favor of specified judgment. Take the data in Table 5 as an example, as it clearly 
shows, there are five experts giving judgement. And MD of one expert, CD of two experts, HD of two experts. Thus according to the percentage, it can obtain:

$\mathrm{D}($ factor $)=(\mathrm{HD}, 0.4)(\mathrm{CD}, 0.4)(\mathrm{MD}, 0.2)$

\begin{tabular}{|c|c|c|c|c|c|}
\hline Expert & Expert1 & Expert2 & Expert3 & Expert4 & Expert5 \\
\hline Level of factor & HD & HD & CD & CD & MD \\
\hline
\end{tabular}

Table 5: The example of judgement on factor

According to the above discussion, the representation of judgement in D numbers is shown in Table 6.

\begin{tabular}{|c|c|c|c|}
\hline AlternativeD numbersFactor & Age & Operation & Time \\
\hline Performer 1 & $(5,0.4)(4,0.6)$ & $(5,0.7)(4,0.3)$ & $(5,1.0)$ \\
\hline Performer 2 & $(5,0.1)(4,0.6)(3,0.3)$ & $(4,0.7)(3,0.3)$ & $(5,0.1)(4,0.9)$ \\
\hline Performer 3 & $(4,0.3)(3,0.7)$ & $(3,0.6)(2,0.4)$ & $(4,0.3)(3,0.4)(2,0.3)$ \\
\hline Performer 4 & $(3,0.6)(2,0.4)$ & $(3,0.1)(2,0.9)$ & $(2,0.4)(1,0.6)$ \\
\hline
\end{tabular}

Table 6: The representation of influential factors in D numbers

The performers are determined by three factors (age, operation, time) jointly, thus this step is to aggregate the D numbers of the influential factors. Using Eqs. (8-13), the result is shown in the Table 7:

\begin{tabular}{|c|c|}
\hline Performer & $D$ numbers \\
\hline Performer A & $\left(4.5, \frac{4.9}{16}\right)\left(4.75, \frac{6}{16}\right)\left(5, \frac{5.1}{16}\right)$ \\
\hline Performer B & $\left(3.5, \frac{5.1}{30}\right)\left(3.75, \frac{6.4}{30}\right)\left(4, \frac{7.3}{30}\right)\left(4.25, \frac{7.7}{30}\right)\left(4.5, \frac{2.2}{30}\right)\left(4.75, \frac{1.3}{30}\right)$ \\
\hline Performer C & $\left(2.25, \frac{2.3}{24}\right)\left(2.5, \frac{3.2}{24}\right)\left(2.75, \frac{4.8}{24}\right)\left(3, \frac{3.6}{24}\right)\left(3.25, \frac{4.8}{24}\right)\left(3.5, \frac{3.2}{24}\right)\left(3.75, \frac{2.1}{24}\right)$ \\
\hline Performer D & $\left(1.5, \frac{3.7}{20}\right)\left(1.75, \frac{4.4}{20}\right)\left(2, \frac{6}{20}\right)\left(2.25, \frac{3.6}{20}\right)\left(2.5, \frac{2.3}{20}\right)$ \\
\hline
\end{tabular}

Table 7: The integration of D numbers: $\quad D_{\text {age }} \oplus D_{\text {operation }} \oplus D_{\text {time }}$

In order to select the optimal performer, given the existed judgement, the I(D) like a kind of utility needed to be calculated as a evaluate criteria by using Eq. (14).

$I\left(D_{\text {performer } A}\right)=4.5 \times \frac{4.9}{16}+4.75 \times \frac{6}{16}+5 \times \frac{5.1}{16}=4.735$

$I\left(D_{\text {performer } B}\right)=3.5 \times \frac{5.1}{30}+3.75 \times \frac{6.4}{30}+4 \times \frac{7.3}{30}+4.25 \times \frac{7.7}{30}+4.5 \times \frac{2.2}{30}+4.75 \times \frac{1.3}{30}=3.995$

$I\left(D_{\text {performer } C}\right)=2.25 \times \frac{2.3}{24}+2.5 \times \frac{3.2}{24}+2.75 \times \frac{4.8}{24}+3 \times \frac{3.6}{24}+3.25 \times \frac{4.8}{24}+3.5 \times \frac{3.2}{24}+3.75 \times \frac{2.1}{24}=2.869$ $I\left(D_{\text {performer } D}\right)=1.5 \times \frac{3.7}{20}+1.75 \times \frac{4.4}{20}+2 \times \frac{6}{20}+2.25 \times \frac{3.6}{20}+2.5 \times \frac{2.3}{20}=1.955$

As shown in Fig. 4, performer A has biggest I(D), which means the worst performer. The assumption in this paper is that the best performer should be with the lowest CHEP. The relationship between I(D) and conditional human error probability (CHEP) is positive correlation, thus we conclude the performer D should have the lowest CHEP. In the next section, the verification will be discussed.

\subsection{Verification}

In the above section, the best performer has been selected. But for verifying our assumption, the final CHEP is necessary. By comparing the conditional human error probability (CHEP), the performer with the lowest CHEP means that performer has lowest probability to make mistakes. 
In reality, the alternative performers will face same problem, under the same environment, at the same time, for which we assume the factors of "closeness in time" and "task relatedness" are same for each case. And the BPA of "similarity of performer" is obtained by Eqs. (15-16) and is illustrated in Table 8 .

\begin{tabular}{|c|c|c|c|}
\hline & Performers & Task & Time \\
\hline $\mathrm{A}$ & $\mathrm{m}(5)=0.7 ; \mathrm{m}(4)=0.3$ & $\mathrm{~m}(5)=0.2 ; \mathrm{m}(4)=0.6 ; \mathrm{m}(3)=0.2$ & $\mathrm{~m}(5)=0.2 ; \mathrm{m}(4)=0.5 ; \mathrm{m}(3)=0.3$ \\
$\mathrm{~B}$ & $m(5)=\frac{0.2}{3} ; m(4)=\frac{2.2}{3} ; m(3)=\frac{0.6}{3}$ & $\mathrm{~m}(5)=0.2 ; \mathrm{m}(4)=0.6 ; \mathrm{m}(3)=0.2$ & $\mathrm{~m}(5)=0.2 ; \mathrm{m}(4)=0.5 ; \mathrm{m}(3)=0.3$ \\
$\mathrm{C}$ & $m(4)=\frac{0.6}{3} ; m(3)=\frac{1.7}{3} ; m(2)=\frac{0.7}{3}$ & $\mathrm{~m}(5)=0.2 ; \mathrm{m}(4)=0.6 ; \mathrm{m}(3)=0.2$ & $\mathrm{~m}(5)=0.2 ; \mathrm{m}(4)=0.5 ; \mathrm{m}(3)=0.3$ \\
$\mathrm{D}$ & $m(3)=\frac{0.7}{3} ; m(2)=\frac{1.7}{3} ; m(1)=\frac{0.6}{3}$ & $\mathrm{~m}(5)=0.2 ; \mathrm{m}(4)=0.6 ; \mathrm{m}(3)=0.2$ & $\mathrm{~m}(5)=0.2 ; \mathrm{m}(4)=0.5 ; \mathrm{m}(3)=0.3$ \\
\hline
\end{tabular}

Table 8: The BPA of different cases

In this paper, it assumes that the weight of factors of same level is same. By using D-S evidence theory Eq. (6), the fused BPA of each factor is obtained as shown in Table 9.

\begin{tabular}{|c|c|c|c|c|}
\hline Case & A & B & C & D \\
\hline & $\mathrm{m}(5)=0.2373$ & $\mathrm{~m}(5)=0.0114$ & $\mathrm{~m}(4)=0.6383$ & $\mathrm{~m}(3)=1$ \\
BBA & $\mathrm{m}(4)=0.7627$ & $\mathrm{~m}(4)=0.9375$ & $\mathrm{~m}(3)=0.3617$ & \\
& & $\mathrm{~m}(3)=0.0511$ & & \\
\hline
\end{tabular}

Table 9: The final result of BPA

Due to the data used in the paper are all single subset as well as $m(\emptyset)=0$, according to the Eq. (7), the numerical value of BetP is equal to the numerical value of BPA. Assume that basic human error probability of the subsequent task $T_{b}$ is $\mathrm{P}(\mathrm{B})=0.01$. Then the conditional human error probability (CHEP) is calculated using Eq. (1), the result is shown in Table 10 and Fig. $4(\mathrm{~b})$.

\begin{tabular}{|c|c|c|c|c|}
\hline Case & A & B & C & D \\
\hline CHEP & 0.6224 & 0.5387 & 0.4337 & 0.2286 \\
\hline
\end{tabular}

Table 10: The final result of CHEP

\subsection{Discussion}

The result of proposed method consists of two parts: the selection of alternatives and the calculation of CHEP. In the first part, the D numbers is used to represent the judgements given by experts. It is reasonable and intelligible way to handle such problem. The number of I(D) is treated as a criteria for performers. The smaller $\mathrm{I}(\mathrm{D})$ is, the better performer is. And the second part discusses the conditional human error probability (CHEP) that means that the failure probability of the following task given the failed preceding task. It is obvious the lower CHEP indicates the better situations. By comparing with Fig. 4, it concludes the relationship between I(D) and CHEP is positive correlation. Thus the gained result complies with our assumption, the performer A selected through proposed method has the highest CHEP 0.6224. It means that the task tends to fail with the most high probability when performer A is selected. $C>D$

$$
I\left(D_{\text {performer } A}\right)>I\left(D_{\text {performer } B}\right)>I\left(D_{\text {performer } C}\right)>I\left(D_{\text {performer } D}\right) C H E P: A>B>
$$



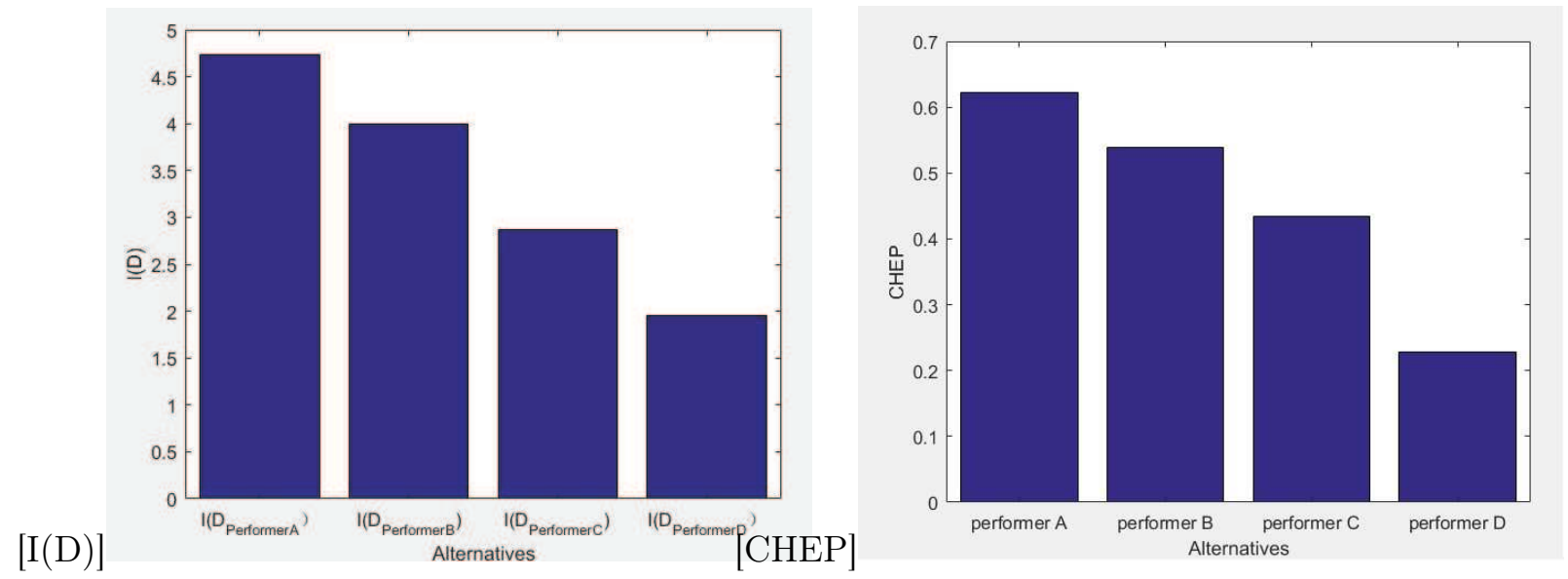

Figure 4: The comparison of ID and CHEP

\section{Conclusion}

In previous works in human reliability analysis (HRA), the influence of performer is not paid enough attention. In this paper, the evaluation method based on $\mathrm{D}$ numbers is presented to deal with the selection of alternative performers in an reasonable and simple way. In the real world, there is no doubt that performers are the main factor that affect our selection. The contribution of this paper is that offering a new way to translate the BPA into D numbers, which is more flexible than traditional ways and reduce the subjectivity at some extent. The final result demonstrates the effectiveness of the proposed method. In the future works, we would take the weights of different influential factors into consideration to make the predicted result more accurate.

\section{Funding}

The work is partially supported by National Natural Science Foundation of China (Grant Nos. 61573290, 61503237).

\section{Conflict of interest}

The authors declare that there is no conflict of interests regarding the publication of this paper.

\section{Bibliography}

[1] Carvalho, P. V.; Dos Santos, I. L.; Vidal, M. C. (2006). Safety implications of cultural and cognitive issues in nuclear power plant operation, Applied Ergonomics, 37(2), 211-223, 2006.

[2] Čepin, M. (2008). Importance of human contribution within the human reliability analysis (ijs-hra), Journal of Loss Prevention in the Process Industries, 21(3), 268-276, 2008. 
[3] Chen, L.; Deng, X. (2018). A modified method for evaluating sustainable transport solutions based on ahp and Dempster-Shafer evidence theory. Applied Sciences, 8(4), Article ID 563, 2018 .

[4] Chen, L.; Deng, Y. (2018). A new failure mode and effects analysis model using DempsterShafer evidence theory and grey relational projection method, Engineering Applications of Artificial Intelligence, 76, 13-20, 2018.

[5] Cooper, S. E.; Ramey-Smith, A.; Wreathall, J.; Parry, G. (1996). A technique for human error analysis (atheana). Technical report, Nuclear Regulatory Commission, Washington, DC (United States). Div. of Systems Technology; Brookhaven National Lab., Upton, NY (United States); Science Applications International Corp., Reston, VA (United States); NUS Corp., Gaithersburg, MD (United States).

[6] Cui, H.; Liu, Q.; Zhang, J.; Kang, B. (2019). An improved deng entropy and its application in pattern recognition, IEEE Access, 7, 18284-18292, 2019.

[7] Dempster, A. P. (2008). Upper and lower probabilities induced by a multivalued mapping, In Classic Works of the Dempster-Shafer Theory of Belief Functions, 57-72. Springer, 2008.

[8] Deng, X.; Jiang, W. (2019). D number theory based game-theoretic framework in adversarial decision making under a fuzzy environment, International Journal of Approximate Reasoning, 106, 194-213, 2019.

[9] Deng, X.; Jiang, W.; Wang, Z. (2019). Zero-sum polymatrix games with link uncertainty: A Dempster-Shafer theory solution, Applied Mathematics and Computation, 340, 101-112, 2019.

[10] Deng, Y. (2012). D numbers: Theory and applications, Journal of Information \& Computational Science, 9(9), 2421-2428, 2012.

[11] Dong, Y.; Zhang, J.; Li, Z.; Hu, Y.; Deng, Y. (2019). Combination of evidential sensor reports with distance function and belief entropy in fault diagnosis, International Journal of Computers Communications \& Control, 14(3), 293-307, 2019.

[12] Dzitac, I.; Filip, F. G.; Manolescu, M.-J. (2017). Fuzzy logic is not fuzzy: World-renowned computer scientist Lotfi A. Zadeh, International Journal of Computers Communications \& Control, 12(6), 748-789, 2017.

[13] Fei, L.; Deng, Y. (2019). A new divergence measure for basic probability assignment and its applications in extremely uncertain environments, International Journal of Intelligent Systems, 34(4), 584-600, 2019.

[14] Fei, L.; Deng, Y.; Hu, Y. (2019). DS-VIKOR: A New Multi-criteria Decision-Making Method for Supplier Selection, International Journal of Fuzzy Systems, 21(1), 157-175, 2019.

[15] Gao, X.; Deng, Y. (2019). The generalization negation of probability distribution and its application in target recognition based on sensor fusion, International Journal of Distributed Sensor Networks, 15(5), 2019.

[16] Gao, X.; Deng, Y. (2019). The negation of basic probability assignment, IEEE Access, 7(1), 10.1109/ACCESS.2019.2901932, 2019.

[17] Gertman, D.; Blackman, H.; Marble, J.; Byers, J.; Smith, C. et al. (2005). The spar-h human reliability analysis method, US Nuclear Regulatory Commission, 2005. 
[18] Guan, X.; Liu, H.; Yi, X.; Zhao, J. (2018). The Improved Combination Rule of D Numbers and Its Application in Radiation Source Identification, Mathematical Problems in Engineering, 2018.

[19] Han, Y.; Deng, Y. (2018). An evidential fractal ahp target recognition method, Defence Science Journal, 68(4), 367-373, 2018.

[20] Han, Y.; Deng, Y. (2019). A novel matrix game with payoffs of Maxitive Belief Structure, International Journal of Intelligent Systems, 34(4), 690-706, 2019.

[21] Jiang, W. (2018). A correlation coefficient for belief functions, International Journal of Approximate Reasoning, 103, 94-106, 2018.

[22] Jiang, W.; Huang, C. (2018). A multi-criteria decision-making model for evaluating suppliers in green SCM, International Journal of Computers Communications \& Control, 13(3), 337$352,2018$.

[23] Jiang, W.; Wang, S. (2017). An uncertainty measure for interval-valued evidences, International Journal of Computers, Communications \& Control, 12(5), 2017.

[24] Kang, B.; Deng, Y.; Hewage, K.; Sadiq, R. (2019). A method of measuring uncertainty for Z-number, IEEE Transactions on Fuzzy Systems, 27(4), 731-738, 2019.

[25] Kang, B.; Zhang, P.; Gao, Z.; Chhipi-Shrestha, G.; Hewage, K.; Sadiq, R. (2019). Environmental assessment under uncertainty using Dempster-Shafer theory and z-numbers, pages Published online, doi: 10.1007/s12652-019-01228-y, 2019.

[26] Kirwan, B. (1996). The validation of three human reliability quantification techniques THERP, HEART and JHEDI: Part 1 - technique descriptions and validation issues, Applied ergonomics, 27(6), 359-373, 1996.

[27] Kontogiannis, T.; Malakis, S. (2009). A proactive approach to human error detection and identification in aviation and air traffic control, Safety Science, 47(5), 693-706, 2009.

[28] Lester, R. K.; McCabe, M. J. (1993). The effect of industrial structure on learning by doing in nuclear power plant operation, The Rand Journal of Economics, 418-438, 1993.

[29] Li, M.; Zhang, Q.; Deng, Y. (2018). Evidential identification of influential nodes in network of networks, Chaos, Solitons \& Fractals, 117, 283-296, 2018.

[30] Li, X.; Chen, X. (2018). D-intuitionistic hesitant fuzzy sets and their application in multiple attribute decision making, Cognitive Computation, 10(3), 496-505, 2018.

[31] Li, Y.; Deng, Y. (2018). Generalized ordered propositions fusion based on belief entropy, International Journal of Computers Communications \& Control, 13(5), 792-807, 2018.

[32] Li, Y.; Deng, Y. (2019). TDBF: Two Dimension Belief Function, International Journal of Intelligent Systems, 34, 10.1002/int.22135, 2019.

[33] Lin, S.; Li, C.; Xu, F.; Liu, D.; Liu, J. (2018). Risk identification and analysis for new energy power system in China based on D numbers and decision-making trial and evaluation laboratory (DEMATEL), Journal of Cleaner Production, 180, 81-96, 2018.

[34] Liu, H.; Dzitac, I.; Guo, S. (2018). Reduction of conditional factors in causal analysis, International Journal of Computers, Communications \& Control, 13(3), 2018. 
[35] Meng, D.; Liu, M.; Yang, S.; Zhang, H.; Ding, R. (2018). A fluid-structure analysis approach and its application in the uncertainty-based multidisciplinary design and optimization for blades, Advances in Mechanical Engineering, 10(6), https://doi.org/10.1177/1687814018783410, 2018.

[36] Pan, L.; Deng, Y. (2018). A New Belief Entropy to Measure Uncertainty of Basic Probability Assignments Based on Belief Function and Plausibility Function, Entropy, 20(11), 842, 2018.

[37] Paté-Cornell, E. (2012). On black swans and perfect storms: risk analysis and management when statistics are not enough, Risk Analysis: An International Journal, 32(11), 1823-1833, 2012 .

[38] Pelusi, D.; Mascella, R.; Tallini, L. (2017). Revised gravitational search algorithms based on evolutionary-fuzzy systems, Algorithms, 10(2), 44, 2017.

[39] Pelusi, D.; Mascella, R.; Tallini, L. (2018). A fuzzy gravitational search algorithm to design optimal iir filters. Energies, 11(4), 736, 2018.

[40] Pelusi, D.; Mascella, R.; Tallini, L.; Nayak, J.; Naik, B.;Abraham, A. (2018). Neural network and fuzzy system for the tuning of gravitational search algorithm parameters, Expert Systems with Applications, 102, 234-244, 2018.

[41] Rong, H.; Ge, M.; Zhang, G.; Zhu, M. (2018). An approach for detecting fault lines in a small current grounding system using fuzzy reasoning spiking neural $\mathrm{P}$ systems, International Journal of Computers Communications \& Control, 13(4), 521-536, 2018.

[42] Shafer, G. (1976). A mathematical theory of evidence, Vol. 42. Princeton University Press, 1976.

[43] Shankar, R.; Choudhary, D.; Jharkharia, S. (2018). An integrated risk assessment model: A case of sustainable freight transportation systems, Transportation Research Part D: Transport and Environment, 63, 662-676, 2018.

[44] Shorrock, S. T.; Kirwan, B. (2002). Development and application of a human error identification tool for air traffic control, Applied ergonomics, 33(4), 319-336, 2002.

[45] Song, Y.; Deng, Y. (2019). A new method to measure the divergence in evidential sensor data fusion, International Journal of Distributed Sensor Networks, 15(4), 2019.

[46] Su, X.; Li, L.; Shi, F.; Qian, H. (2018). Research on the fusion of dependent evidence based on mutual information, IEEE Access, 6, 71839-71845, 2018.

[47] Su, X.; Mahadevan, S.; Xu, P.; Deng, Y. (2015). Dependence assessment in human reliability analysis using evidence theory and AHP, Risk Analysis, 35(7), 1296-1316, 2015.

[48] Sun, R.; Deng, Y. (2019). A new method to identify incomplete frame of discernment in evidence theory, IEEE Access, 7(1), 15547-15555, 2019.

[49] Sun, R.; Deng, Y. (2019). A new method to determine generalized basic probability assignment in the open world, IEEE Access, 7(1), 52827-52835, 2019.

[50] Swain, A. (1964). Therp technique for human error rate prediction, In Proceedings of the Symposium on Quantification of Human Performance, Albuquerque, 1964. 
[51] Swain, A. D. (1990). Human reliability analysis: Need, status, trends and limitations, Reliability Engineering Ėamp; System Safety, 29(3), 301-313, 1990.

[52] Wang, J.; Qiao, K.; Zhang, Z. (2019). An improvement for combination rule in evidence theory, Future Generation Computer Systems, 91, 1-9, 2019.

[53] Xiao, F. (2018). A hybrid fuzzy soft sets decision making method in medical diagnosis, IEEE Access, 6, 25300-25312, 2018.

[54] Xiao, F. (2018). A novel multi-criteria decision making method for assessing health-care waste treatment technologies based on D numbers, Engineering Applications of Artificial Intelligence, 71(2018), 216-225, 2018.

[55] Xiao, F. (2019). Multi-sensor data fusion based on the belief divergence measure of evidences and the belief entropy, Information Fusion, 46, 23-32, 2019.

[56] Xiao, F. (2019). A multiple criteria decision-making method based on D numbers and belief entropy, International Journal of Fuzzy Systems, DOI: 10.1007/s40815-019-00620-2, 2019.

[57] Xiao, F.; Ding, W. (2019). Divergence measure of Pythagorean fuzzy sets and its application in medical diagnosis, Applied Soft Computing, 254-267, 2019.

[58] Xu, H.; Deng, Y. (2019). Dependent Evidence Combination Based on DEMATEL Method, International Journal of Intelligent Systems, 1555-1571, 2019.

[59] Yager, R. R. (2012). On z-valuations using zadeh's z-numbers, International Journal of Intelligent Systems, 27(3), 259-278, 2012.

[60] Yager, R. R. (2018). Fuzzy rule bases with generalized belief structure inputs, Engineering Applications of Artificial Intelligence, 72, 93-98, 2018.

[61] Yager, R. R.; Alajlan, N. (2017). Maxitive Belief Structures and Imprecise Possibility Distributions, IEEE Transactions on Fuzzy Systems, 25(4), 768-774, 2017.

[62] Yang, H.; Deng, Y.; Jones, J. (2018). Network division method based on cellular growth and physarum-inspired network adaptation. International Journal of Unconventional Computing, 13(6), 477-491, 2018.

[63] Yin, L.; Deng, Y. (2018). Toward uncertainty of weighted networks: An entropy-based model, Physica A: Statistical Mechanics and its Applications, 508, 176-186, 2018.

[64] Zadeh, L. A. (1965). Fuzzy sets. Information and control, 8(3), 338-353, 1965.

[65] Zadeh, L. A. (2019). Preliminary draft notes on a similarity-based analysis of time-series with applications to prediction, decision and diagnostics, International Journal of Intelligent Systems, 34(1), 107-113, 2019.

[66] Zheng, X.; Deng, Y. (2018). Dependence assessment in human reliability analysis based on evidence credibility decay model and iowa operator, Annals of Nuclear Energy, 112, 673-684, 2018.

[67] Zhou, M.; Liu, X.-B.; Chen, Y.-W.; Yang, J.-B. (2018). Evidential reasoning rule for MADM with both weights and reliabilities in group decision making, Knowledge-Based Systems, 143, 142-161, 2018. 
[68] Zhou, M.; Liu, X.-B.; Yang, J.-B.; Chen, Y.-W.; Wu, J. (2019). Evidential reasoning approach with multiple kinds of attributes and entropy-based weight assignment, KnowledgeBased Systems, 163, 358-375, 2019.

[69] Zhou, M.; Liu, X.; Yang, J. (2017). Evidential reasoning approach for MADM based on incomplete interval value, Journal of Intelligent \& Fuzzy Systems, 33(6), 3707-3721, 2017.

[70] Zhou, X.; Hu, Y.; Deng, Y.; Chan, F, T. S.; Ishizaka, A. (2018). A DEMATEL-Based Completion Method for Incomplete Pairwise Comparison Matrix in AHP, Annals of Operations Research, 271(2), 1045-1066, 2018.

[71] Zio, E. (2009). Reliability engineering: Old problems and new challenges, Reliability Engineering \& System Safety, 94(2), 125-141, 2009.

[72] Zio, E.; Baraldi, P.; Librizzi, M.; Podofillini, L.; Dang, V. N. (2009). A fuzzy set-based approach for modeling dependence among human errors, Fuzzy Sets and Systems, 160(13), 1947-1964, 2009. 